\title{
Trends in Medicine
}

\section{Opinion}

\section{Complementary and alternative medicine-use and potential interactions}

\section{Bimal Roy Krishna* \\ College of Osteopathic Medicine, Touro University, USA}

The use and popularity of complementary and alternative medicine (CAM) has sharply risen over the decades particularly in the United States. It is postulated that up to $20 \%$ of American adults use at least one herbal product on a weekly basis. During pregnancy and lactation up to $15 \%$ of patients use herbal products for various reasons.

This includes herbal medications and practicing yoga, ayurvedic medicine, acupuncture and various others. It is postulated that there are several reasons including cost, perceived data, enhanced effects and non-regulated. Many medical schools are now introducing this into their curriculum to raise awareness.

CAM is not FDA approved and the complete active ingredients are unknown as is the pharmacokinetic disposition and mechanism of action. Literature speculates on the mechanism of action. Chronic use particularly in geriatric patients and with prescribed medications poses risks, side effects and potential interactions some of which are known. This may be exacerbated in patients with hepatic and renal compromise. Contraindications and use in pregnancy and pediatric patients is unclear. It is clear that in pregnancy and lactation there is no clear and documented benefit. These products are also widely used in terminal illnesses.
Copyright: C2017 Krishna BR. This is an open-access article distributed under the terms of the Creative Commons Attribution License, which permits unrestricted use, distribution, and reproduction in any medium, provided the original author and source are credited.
Numerous side effects and drug interactions have been documented. Some potential concerns and side effects are the serotonin syndrome, induction of the CYP450 system, bleeding and gastrointestinal disorders and rhabdomyolysis. This also extends to potential interactions with prescribed medication and attenuation of side effects.

It is speculated that a number of herbal products influence physiological processes and mechanism and affect homeostasis. To name a few, platelet aggregation, alpha 1 receptor blockade, vasomotor tone, free radical scavenging and neurotransmitter uptake. These effects can have profound physiological consequences and drug interactions are of immediate concern.

It is evident that a number of practitioners encourage the use of herbal products however since the pharmacokinetic disposition is unknown extreme caution must be taken in these circumstances. Polypharmacy always poses the risk of adverse drug reactions and interactions.

As part of the patient history, herbal medication consumption should be documented and its cessation encourage prior to prescribing any medication/s.
Correspondence to: Bimal Roy Krishna, Professor \& Director of Pharmacology, College of Osteopathic Medicine, Touro University, USA, E-mail: BimalRoy. Krishna@tun.touro.edu

Received: November 01, 2017; Accepted: November 16, 2017; Published: November 20, 2017 